\title{
Single-Molecule Photoswitching Enables High-Resolution Optical Imaging
}

\author{
Dehong Hu*, Zhiyuan Tian**, Wuwei Wu**, Wei Wan**, Alexander D. Q. Li** \\ *Pacific Northwest National Laboratory, PO Box 999, Richland, WA 99352 \\ **The Department of Chemistry, Washington State University, Pullman, WA 99164
}

Fluorescence imaging of biological systems with spatial resolution beyond diffraction limit is of intensive interests. Recently, it has been discovered that by stochastically photoswitching fluorophores on and off, the diffraction limit can be circumvented in far-field optical imaging.[1][2] Here we present a small photoswitching dye, spiropyran derivatives (Fig. 1) with the desired characteristics as the excellent photoswitchable molecular fluorophores.[3] Using such a fluorophore, we have achieved photoactuated unimolecular logical switching attained reconstruction (PULSAR) microscopy, with resolution down to 10-40 nm, far beyond the diffraction limit. Spiropyran requires no special treatment to cells and can be readily modified using chemical functional groups for specific targeting.

Spiropyran has no fluorescence under visible excitation. However, upon UV excitation, spiropyran changes to merocyanine dye. Merocyanine produces strong red-fluorescence under visible excitation. We explored the single-molecule photophysics of merocyanine. Spin-coating dilute spiropyran and poly (methyl methacrylate) in dichloromethane resulted in thin films containing isolated photoswitchable dyes. Next, the spiropyran was switched to the merocyanine fluorophore by UV illumination. Under 532-nm excitation, the single-molecule fluorescence from merocyanine was detected. A representative fluorescence intensity trajectory is shown in Fig. 1 and it exhibits typical single-molecule signatures such as intensity blinking and one-step photobleaching.

To demonstrate PULSAR microscope capability, we imaged nanoparticles in HeLa cells. The nanoparticles containing spiropyran were delivered and transported into lysosomes. Then we fixed the HeLa cells, selected a sub-cellular area of interest (Fig. 2a), zoomed into this region (Fig. 2b\&c), and collected both white light images and conventional fluorescence imaging. The residual fluorescence from interfering fluorophores and background were pre-photobleached by 532-nm light. PULSAR microscopy alternated a controlled-power UV on-switching pulse and a 532-nm probe pulse. The UV pulses only switch on less than one molecule per nanoparticle on average for each pulse. This pulse-and-probe pattern repeated many cycles to accumulate hundreds of singlemolecule emission images. The single-molecule locations were extracted from the images and were reconstructed to the final high-resolution image. Fig. 3d displays the PULSAR images of the nanoparticles in a lysosome of a fixed cell. The nanoparticles were resolved inside a single organelle by PULSAR while traditional wide field optical microscopy cannot resolve multiple nanoparticles residing in a lysosome due to the diffraction limit.

\section{References}

[1] E. Betzig et al, Science 313 (2006) 1642

[2] M. J. Rust et al, Nat Meth 3 (2006) 793

[3] L.Y. Zhu et al, J. Am. Chem. Soc. 129 (2007) 3524

[4] This work was supported by EMSL, a DOE user facility and a grant from NIH 

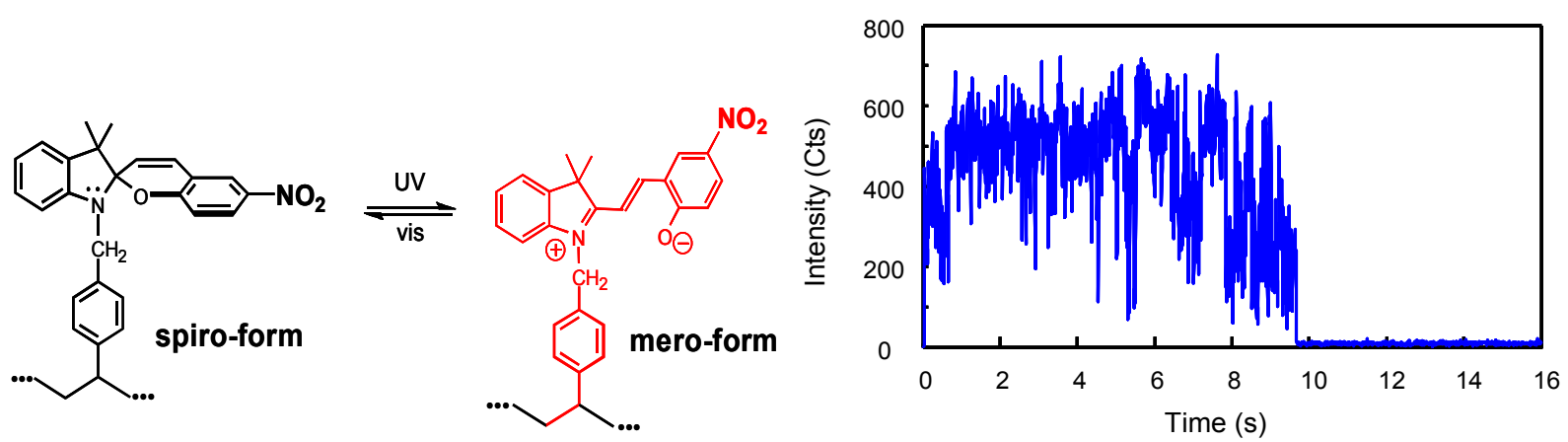

Fig. 1 (Left) Spiropyran (spiro-form) and merocyanine (mero-form) were polymerized into the hydrophobic cores of polymer nanoparticles and thus their photochemical conversion occurs within isolated protected nanoparticles. (Right) A typical single-molecule intensity trajectory illustrates that the photoswitching dye in its emissive state-merocyanine behaves very much like normal highquantum yield dyes such as rhodamine.

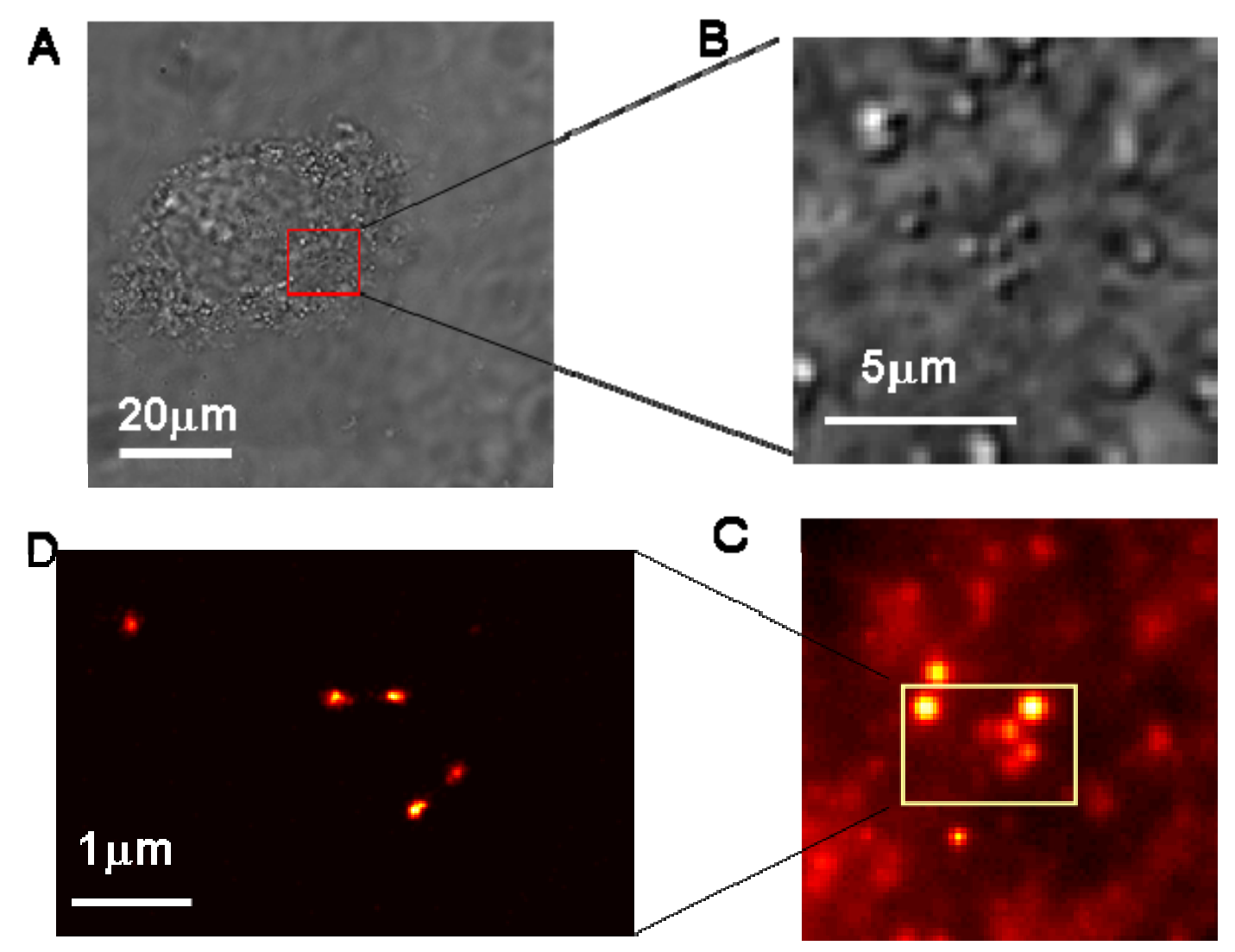

Fig. 2 PULSAR images detected spiropyran-nanoparticles in a fixed cellular organelle. Bright field image of a fixed HeLa cell (A) provided an area of interest (red box). (B) The zoomed-in white-light image indicated nanostructures scattering light. (C) Conventional wide-field fluorescence imaging revealed that some of spots were nanoparticles. (D) The PULSAR image of these spiropyrannanoparticles produced much higher resolution, separating fuzzy clusters into resolved bright spots. 